\title{
Correction
}

\section{Correction: Milivojevic, et al., "Coding of Event Nodes and Narrative Context in the Hippocampus"}

In the article "Coding of Event Nodes and Narrative Context in the Hippocampus" by Branka Milivojevic, Meryl Varadinov, Alejandro Vicente Grabovetsky, Silvy H. P. Collin, and Christian F. Doeller, which appeared on pages 12412-12424 of the December 7, 2016 issue, there were two instances of the same typo which resulted in accidentally switching labels 'Within' and 'Across' in Table 2, which appeared on page 12418 and again on page 12420 in the Results section. The corrected sentence in the Results section on line 19 is as follows: ". . . there were no differences in hippocampal pattern similarity using the Across-Narrative-Character-Networks or AcrossNarrative-Location-Networks contrasts, ...”. This correction does not affect the conclusions of the paper. Table 2 has been corrected on the online PDF version and displayed below.

Table 2. Summary of representational similarity results for the control analyses ${ }^{a}$

\begin{tabular}{|c|c|c|c|c|c|c|}
\hline \multirow[b]{2}{*}{ Analysis } & \multirow[b]{2}{*}{ Region } & \multirow[b]{2}{*}{ Laterality } & \multicolumn{3}{|c|}{ MNI coordinates } & \multirow[b]{2}{*}{ zScore } \\
\hline & & & $x$ & $y$ & z & \\
\hline Character co-occurrence & Fusiform gyrus & $\mathrm{R}$ & 30 & -56 & -14 & 4.59 \\
\hline \multirow[t]{7}{*}{ Character-Location co-occurrence } & Middle occipital gyrus & $\mathrm{L}$ & -44 & -76 & 10 & 4.61 \\
\hline & Middle temporal gyrus & $\mathrm{L}$ & -46 & -56 & 12 & 4.01 \\
\hline & Middle temporal gyrus & $\mathrm{R}$ & 50 & -58 & 22 & 4.39 \\
\hline & Superior temporal gyrus & $\mathrm{R}$ & 62 & -48 & 22 & 4.09 \\
\hline & Precuneus & $\mathrm{R}$ & 6 & -68 & 54 & 3.38 \\
\hline & Superior parietal & $\mathrm{R}$ & 24 & -76 & 52 & 3.37 \\
\hline & Angular gyrus & $\mathrm{R}$ & 38 & -72 & 40 & 3.31 \\
\hline \multirow[t]{2}{*}{ Across-Narrative Location Networks } & Inferior frontal gyrus & $\mathrm{L}$ & -36 & 24 & 20 & 4.08 \\
\hline & Superior frontal gyrus & $\mathrm{L}$ & -16 & 26 & 44 & 3.27 \\
\hline \multirow[t]{6}{*}{ Across-Narrative Character Networks } & Caudate & $\mathrm{L}$ & -8 & 10 & 16 & 5.47 \\
\hline & Thalamus & $\mathrm{R}$ & 8 & -20 & 6 & 4.17 \\
\hline & Middle cingulate & $\mathrm{L}$ & -4 & -34 & 48 & 3.98 \\
\hline & Dorsal ACC & $\mathrm{R}$ & 4 & -40 & 38 & 3.67 \\
\hline & Posterior cingulate & $\mathrm{R}$ & 8 & -40 & 18 & 3.45 \\
\hline & Putamen & $\mathrm{R}$ & 24 & 8 & 8 & 3.95 \\
\hline \multirow[t]{14}{*}{ Within-Narrative Locations } & Lingual gyrus & L & -20 & -80 & -8 & 5.01 \\
\hline & Cerebellum & L & -10 & -74 & -22 & 4.48 \\
\hline & Fusiform & L & -28 & -32 & -18 & 4.45 \\
\hline & Hippocampus & L & -20 & -36 & 4 & 4.11 \\
\hline & Fusiform & $\mathrm{R}$ & 36 & -54 & -10 & 4.44 \\
\hline & Thalamus & $\mathrm{R}$ & 20 & -26 & 12 & 3.87 \\
\hline & Hippocampus & $\mathrm{R}$ & 28 & -30 & 4 & 3.79 \\
\hline & Putamen & $\mathrm{L}$ & -28 & 0 & 2 & 3.93 \\
\hline & Lingual gyrus & $\mathrm{R}$ & 24 & -86 & 0 & 3.55 \\
\hline & Calcarine & $\mathrm{R}$ & 24 & -60 & 14 & 3.55 \\
\hline & Precuneus & $\mathrm{R}$ & 14 & -50 & 14 & 3.39 \\
\hline & Cerebellum & $\mathrm{R}$ & 24 & -74 & -24 & 3.5 \\
\hline & Calcarine & $\mathrm{L}$ & -2 & -86 & 12 & 3.38 \\
\hline & Cerebellum & $\mathrm{R}$ & 24 & -60 & -48 & 3.12 \\
\hline \multirow[t]{7}{*}{ Within-Narrative Characters } & Cuneus & $\mathrm{R}$ & 12 & -86 & 18 & 5.43 \\
\hline & Superior occipital gyrus & $\mathrm{R}$ & 22 & -88 & 6 & 5.3 \\
\hline & Calcarine & $\mathrm{R}$ & 16 & -44 & 6 & 4.68 \\
\hline & Hippocampus & $\mathrm{R}$ & 38 & -32 & -6 & 4.6 \\
\hline & Cerebellum & $\mathrm{L}$ & -18 & -72 & -20 & 3.62 \\
\hline & Middle occipital gyrus & $\mathrm{L}$ & -18 & -96 & 10 & 3.26 \\
\hline & Lingual gyrus & $\mathrm{L}$ & -16 & -90 & 0 & 2.72 \\
\hline
\end{tabular}

${ }^{a}$ Character co-occurrence and Character-Location co-occurrence (see contrasts in Fig. 2D); Within-story Locations and Within-story Characters (see contrasts in Fig. 2E); and Across-story Locations and Characters (see contrasts in Fig. 2F). Reported statistics are for peak voxels $8 \mathrm{~mm}$ apart within clusters significant at corrected statistical threshold of $p<0.05$ on the cluster-level.

DOI: 10.1523/JNEUROSCI.1159-17.2017 